\title{
A PRACTICAL EFFECT OF THE RECENT O16 CROSS SECTION AND MUBAR UNCERTAINTIES
}

\author{
D. Kent Parsons ${ }^{1}$, Scott A. Turner ${ }^{1}$, and Peter J. Jaegers ${ }^{1}$ \\ ${ }^{1}$ Los Alamos National Laboratory \\ Los Alamos NM United States of America 87544
}

dkp@lanl.gov, turners@lanl.gov,pjaegers@lanl.gov

\begin{abstract}
With the recent release of ENDF/B VIII.0 data, additional covariance data was provided for many isotopes, including O16. The detail of elastic scattering and mubar covariance data for O16 increased dramatically between ENDF/B VII.1 and ENDF/B VIII.0. This new covariance data has been processed with NJOY2016 and investigation has begun on the effects of these new uncertainty data. The uncertainties are applied to multi-group scattering cross sections and P1 Legendre components in deterministic neutron transport.

A simple but typical application of shielding fission neutrons with concrete has been used to assess the practical effects of the new covariance data for O16. A somewhat surprising result is that the mubar uncertainty can have a significant effect on the calculated shielding and criticality results.
\end{abstract}

KEYWORDS: uncertainty, sensitivity, mubar

\section{Introduction}

In recent years, nuclear data evaluation files have included more uncertainty information in the form of covariance data on the cross sections. While the reaction rate cross sections can be found in section MF 3 of the ENDF- 6 format $^{1}$, the related covariance data can be found in section MF 33. Very recently, covariance data on mubar (i.e., the P1/P0 ratio for elastic scattering) has begun to appear in the evaluation files as well. Since the angular distribution information for elastic scattering is usually found in section MF 4, the associated covariance data is found in section MF 34.

The effect of mubar on multi-group transport calculations is manifested as how forward (in the lab frame) or how backward (in the lab frame) the elastic scattering is. More forward scattering tends to increase neutron leakage, while more backward scattering decreases neutron leakage. So unlike cross section uncertainties which interact with the P0 moment fluxes, the mubar uncertainties interact with the P1 moment fluxes. 
In the initial ENDF/B VIII nuclear data release, covariance data for mubar is given for U-235, $\mathrm{Pu}-239$, and $\mathrm{O}-16$, among others. This mubar data for $\mathrm{Pu}-239$ and $\mathrm{O}-16$ will be investigated in this paper and applied to selected test problems.

The processing and application of this mubar data involves several procedures depending on the desired analysis. The first step is to process the covariance data in the evaluation file into a multi-group covariance matrix using a data processing code like $\mathrm{NJOY}^{2}$.

If the desired uncertainty is for the calculated $k_{\text {eff }}$ of a static critical assembly, then the covariance matrix may be combined with appropriate sensitivity vectors in the "sandwich" rule.

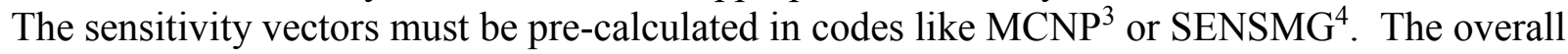
uncertainty of $\mathrm{k}_{\mathrm{eff}}$ comes out of the "sandwich" rule multiplication.

If the desired uncertainty is for a complex dynamic application, then the "sandwich" rule may not be appropriate. Thus, the covariance data is used to generate replica input data for multiple calculational runs. The outputs from each of the multiple runs are then tabulated to measure the uncertainty of the desired outputs with respect to the input variations.

\subsection{Previous Work}

Previous work ${ }^{5,6,7}$ along these lines has been carried out with arbitrary estimated covariance data for mubar. The published results suggest that "mubar" covariance data can be an important source of uncertainty for small problems with a relatively high percentage of neutron leakage. The previous work has also been limited to "sandwich" rule applications. The work presented here uses "real" mubar covariance data and also includes applications for which the "sandwich" rule is not appropriate.

\section{Processing the mubar Covariance Data into Multi-group Matrices}

NJOY modules ERRORR, COVR, and VIEWR are used to process the mubar covariance data from the evaluation files. The procedure is similar to processing cross section covariance data.

Covariance matrices can be calculated in either "relative" (with respect to the mean values of mubar) or "absolute" form. The relative covariance matrices are often used in the "sandwich" rule when the sensitivities are given in (say, $\% \mathrm{k}_{\text {eff }} / \% \mathrm{xs}$ ) units, while the absolute covariances are conveniently used in the generation of replica data.

In general, covariance matrices are symmetric and positive (or semi-positive) definite. Positive (or semi-positive) definite matrices have positive (or non-negative) eigenvalues. A practical problem which often arises in the generation of these multi-group covariance matrices is that "small" negative eigenvalues are generated. "Small" is loosely defined as having an absolute value significantly less than the large positive eigenvalues. These "small" negative eigenvalues are due to the (effectively) single-precision data limitations of the ENDF-6 format. Beware that canned software subroutines for manipulating covariance matrices often cannot handle these negative eigenvalues. 
In Table I, a typical sample set of 30 eigenvalues from a covariance matrix is given to illustrate this problem.

Table I. Typical Mubar Covariance Eigenvalues

\begin{tabular}{|c|c|c|c|c|}
\hline 0.0012139 & $3.9759 \mathrm{E}-06$ & $2.6692 \mathrm{E}-08$ & 0 & 0 \\
\hline 0.00051391 & $5.7225 \mathrm{E}-07$ & $1.3939 \mathrm{E}-08$ & 0 & $-2.5856 \mathrm{E}-12$ \\
\hline 0.00014548 & $9.5012 \mathrm{E}-08$ & $7.03 \mathrm{E}-09$ & 0 & $-2.1816 \mathrm{E}-10$ \\
\hline 0.00003267 & $5.4728 \mathrm{E}-08$ & $2.6692 \mathrm{E}-08$ & 0 & $-5.9191 \mathrm{E}-09$ \\
\hline 0.000013658 & $4.8051 \mathrm{E}-08$ & $1.3939 \mathrm{E}-08$ & 0 & $-1.6388 \mathrm{E}-08$ \\
\hline $9.4226 \mathrm{E}-06$ & $4.0415 \mathrm{E}-08$ & $7.03 \mathrm{E}-09$ & 0 & $-5.5935 \mathrm{E}-08$ \\
\hline
\end{tabular}

As long as the negative eigenvalues are "small" (like the last 5 eigenvalues shown above), then either of two practical approaches can be used in the generation of replica data. First, replica data can be generated using only the positive eigenvalues and ignoring the zero and "small" negative eigenvalues. This is the approach taken in this work. The rationale is that the zero eigenvalues and their eigenvectors and the "supposed to be zero but small negative" eigenvalues and their eigenvectors do not really contribute very much to the behavior of the covariance matrix.

Second, as an alternative approach, a "small" ridge correction can be added to the diagonal terms of the covariance matrix (say a value of $5.5935 \mathrm{e}-8$ ). This increases the value of all eigenvalues by the value of the correction. While this approach will eliminate all negative eigenvalues, it will also change the values of the previously zero and negative eigenvalues and their associated eigenvectors significantly. However, this approach has only a small effect on the dominant larger positive eigenvalues and their eigenvectors.

If the negative eigenvalues from a covariance matric are not "small", then the covariance matrix is invalid and should be discarded.

As given in ENDF/B-VIII data and processed by NJOY, mubar covariance data includes the mean values for mubar at each energy group, the variance (or standard deviation) of mubar at each energy group and also covariance cross terms linking the different groups. There are, at present, no cross terms for mubar across isotopes.

Mubar covariance matrices in 250 groups have been generated for $\mathrm{Pu}-239$ and $\mathrm{O}-16$ from ENDF/B-VIII data. At present, NJOY is unable to process the U-235 data due to the extended formats used in the ENDF/B-VIII evaluation file. This NJOY capability will be generalized in the near future. O-16 mubar covariance data from JENDL-4.0 was also processed due to the problems encountered with the O-16 mubar covariance data from ENDF/B-VIII. Work is currently ongoing at Los Alamos to remedy the O-16 mubar covariance data.

\subsection{Problems with the O-16 mubar Covariance Data currently in ENDF/B-VIII}

As initially given in ENDF/B-VIII, the O-16 covariance data for mubar has some problems. These were first reported by Pino Palmiotti at the November 2018 CSEWG meeting at 


\section{Uncertainties"}

Brookhaven National Laboratory. Specifically, the mubar uncertainties are way too large at lower neutron energies. Another limitation of the Los Alamos O-16 mubar covariance data is that the data only extends to $7 \mathrm{MeV}$. R-matrix theory calculations at higher energies (with more reaction channels) become very difficult. Finally, there are some large unphysical uncertainties at a few specific resonance energies including the oxygen window at $\sim 2.35 \mathrm{MeV}$.

This O-16 data and its problems can be seen in Figure 1. The mubar quantities are tied to the left hand side axis, while the cross sections $(\mathrm{mt} 2)$ are tied to the right hand side axis.

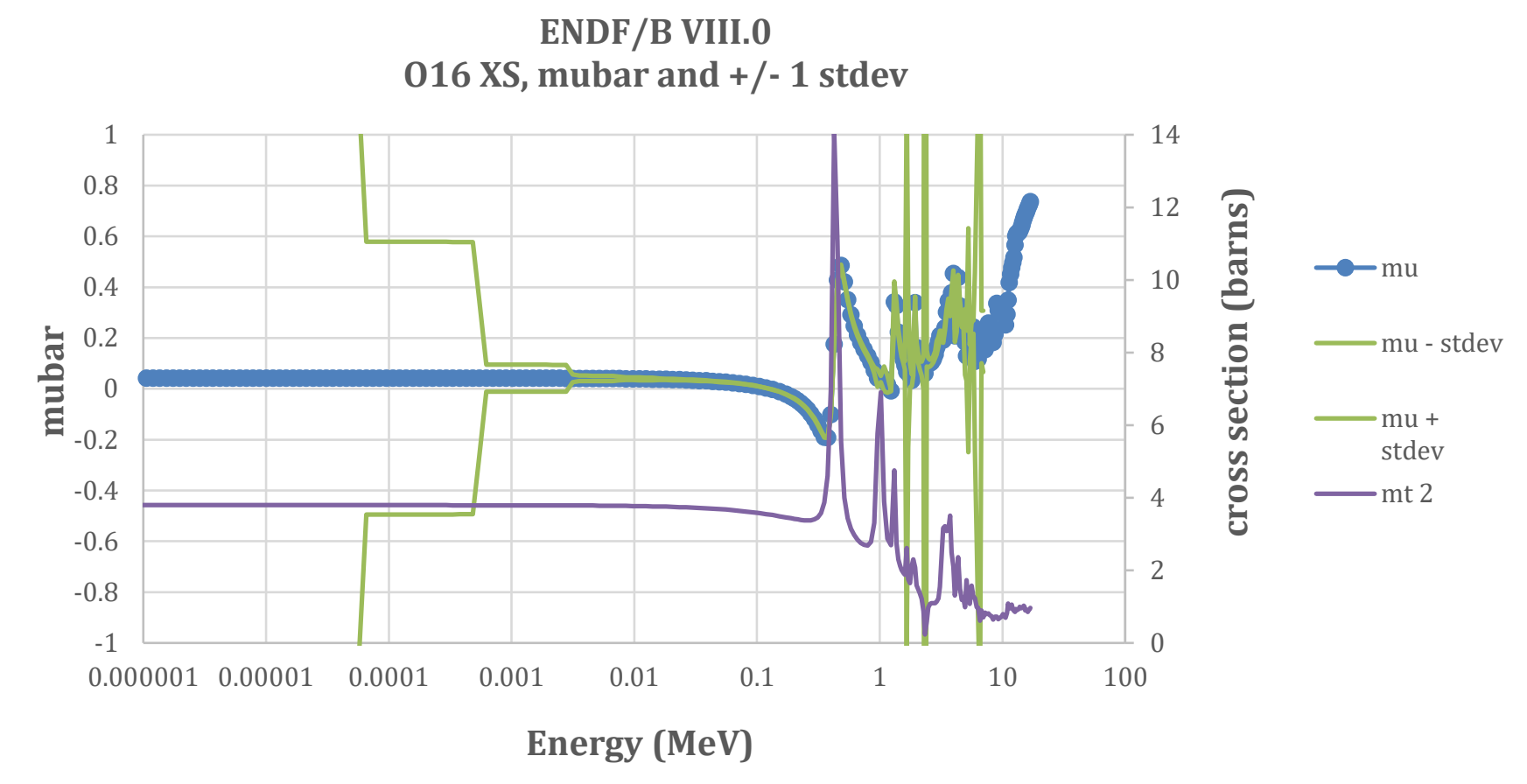

Figure 1. ENDF/B VIII.0 O-16 mubar Data and its Uncertainties

In lieu of the ENDF/B VIII.0 O-16 covariances, the JENDL 4.0 O-16 evaluation was processed into 250 groups for scattering ( $\mathrm{mt} \mathrm{2}$ ) and mubar covariances ( $\mathrm{mf} \mathrm{34}$ ). The mubars and elastic scattering cross sections are very comparable to ENDF/B VIII.0. In contrast with ENDF/B VIII.0, however, the JENDL mubar uncertainties remain bounded, both at lower energies and also at specific higher energy resonance points. This is seen in Figure 2.

\section{Generating Sensitivity Vectors for Use in the Sandwich Rule}

Either MCNP or SENSMG may be used to calculate sensitivity vectors for use in the sandwich rule. For keff problems, the "ksen" option of MCNP may be used. For fixed source problems, the PERT perturbation option may be used ${ }^{8}$. SENSMG may be used for either $k_{\text {eff }}$ or fixed source problems. In either case, the sensitivities are given in units of $(\% / \%)$. 


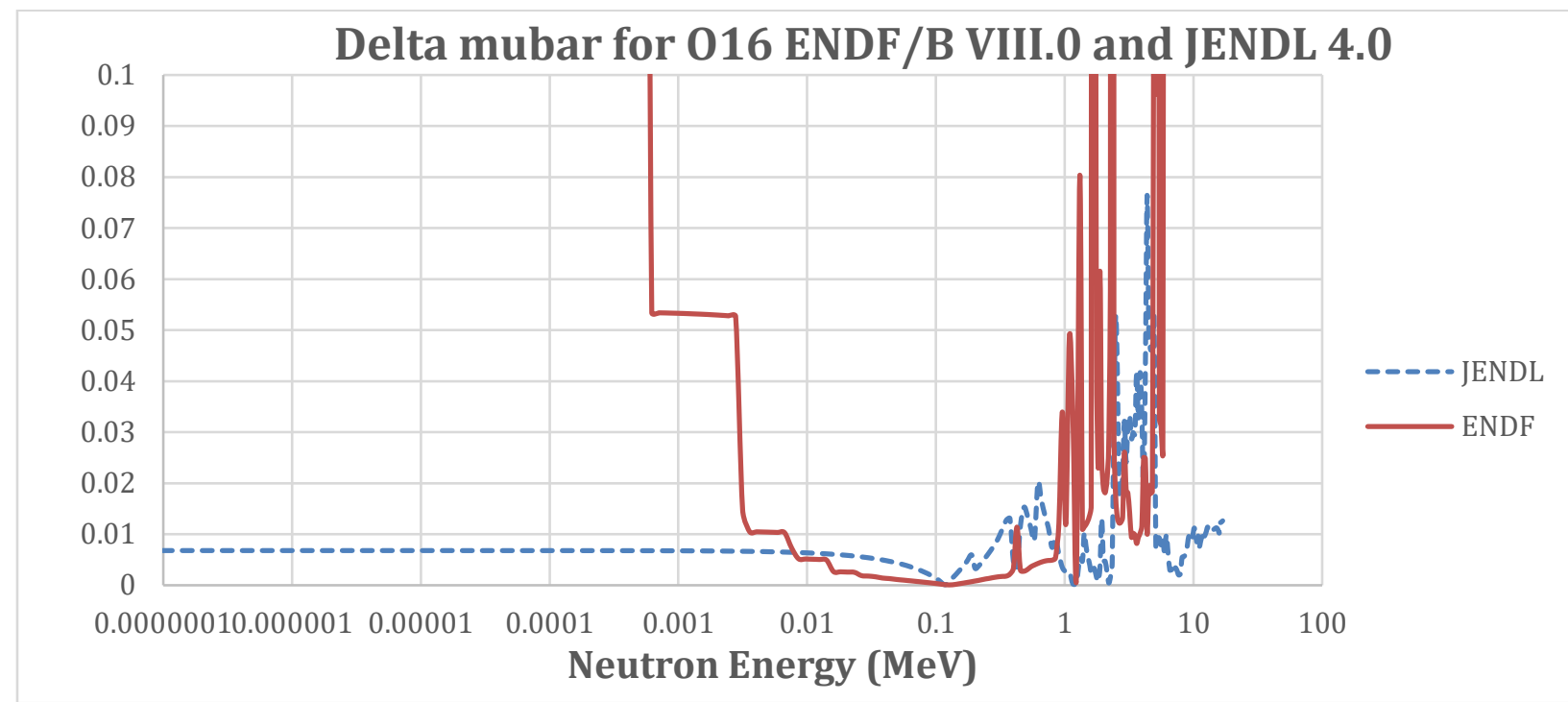

Figure 2. Comparison of mubar Uncertainties Between ENDF/B VIII.0 and JENDL 4.0 for O-16.

Mubar covariances require the use of the P1 fluxes. Elastic scattering PL flux sensitivity vectors can be calculated with MCNP using a feature recently developed ${ }^{6,10,11}$ but the option has not yet been fully vetted. SENSMG can also calculate sensitivities to the P0 and the PL fluxes.

Unfortunately, these quantities are based on the typical deterministic transport scattering blocks and include not only elastic scattering, but inelastic scattering and $(\mathrm{n}, \mathrm{xn})$ reactions as well. For O-16, however, elastic scattering is the dominant contribution in the Legendre scattering block of SENSMG

Currently, SENSMG is unable to give a breakdown of transport scattering block component pieces. The only scattering equivalence discovered so far between higher Legendre order flux sensitivity vectors calculated by SENSMG and the total flux based sensitivities from MCNP is that the sum of the MCNP sensitivity vectors for $m t 2,4,16,17$, etc. is equal to the sum of the SENSMG sensitivity vectors for P0, P1, P2, and P3.

This equivalence is shown in Figure 3. The problem is leakage calculation from a fixed source of neutrons (in a fission spectrum) at the center of a 6 " radius sphere of concrete.

The Pn sensitivity vector elements for each group taken from SENSMG are the sum of the selfscattering plus the out-scattering sensitivities for that group. Even though specific group to specific group sensitivities are available from SENSMG, they are not used here because the mubar covariance data is dimensioned only by the incoming group (i.e., the outgoing group sensitivities are summed together in the covariance data).

Figure 4 compares the $\mathrm{P} 0$ and the $\mathrm{P} 1$ sensitivities for elastic scattering in $\mathrm{O}-16$ for this same shielding problem. Notice that while the P0 sensitivity is generally negative (more elastic scattering leads to less leakage), the P1 sensitivity is generally positive (more forward scattering leads to more leakage - especially at higher neutron energies). 


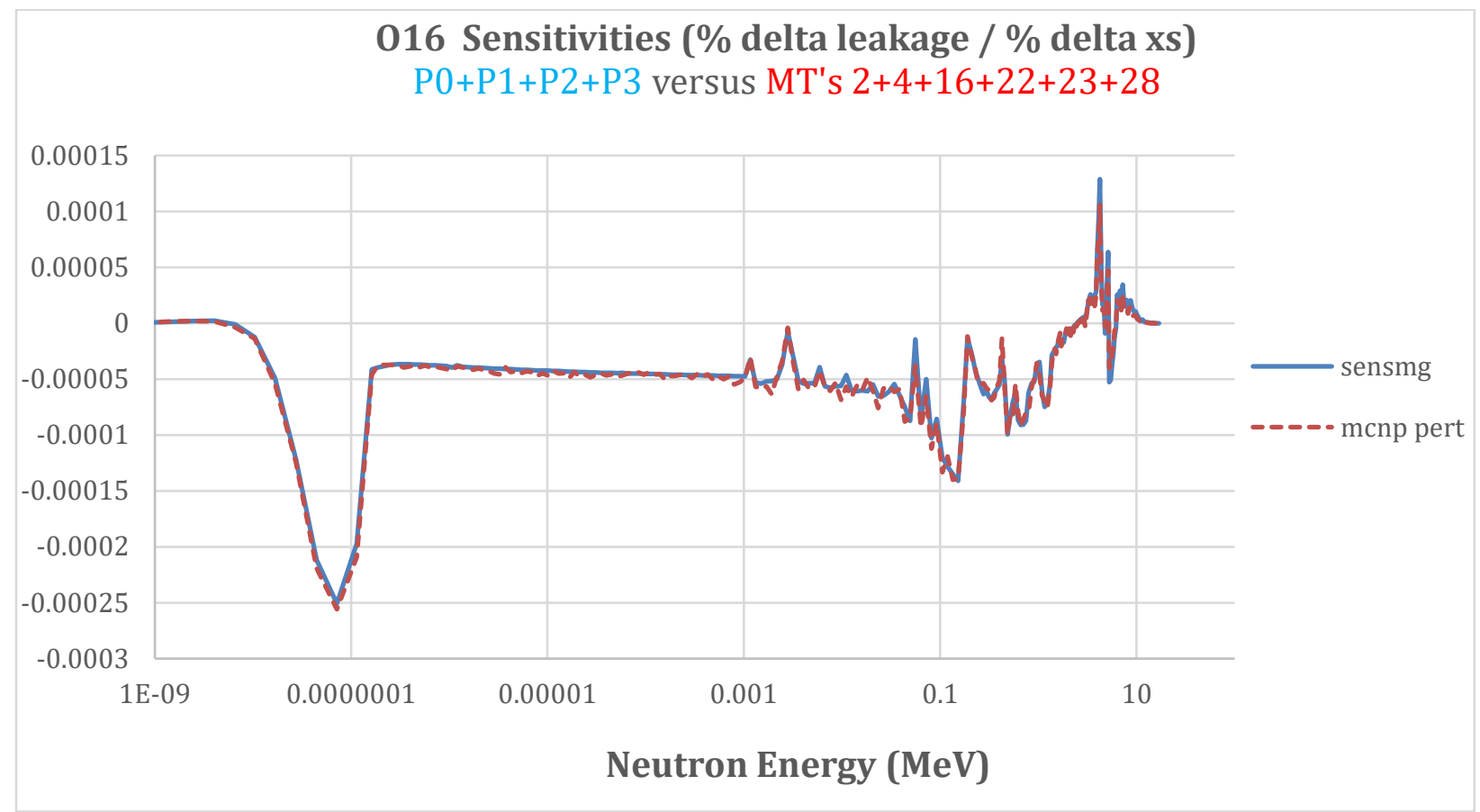

Figure 3. Equivalence of Sensitivities Calculated by MCNP and SENSMG

To apply the sandwich rule, the relative covariance matrix is used to multiply the sensitivity vectors. The final units should come out as the variance / (output) ${ }^{2}$. The square root of this term is thus the standard deviation/output -- which is the definition of the relative standard deviation of the desired output quantity.

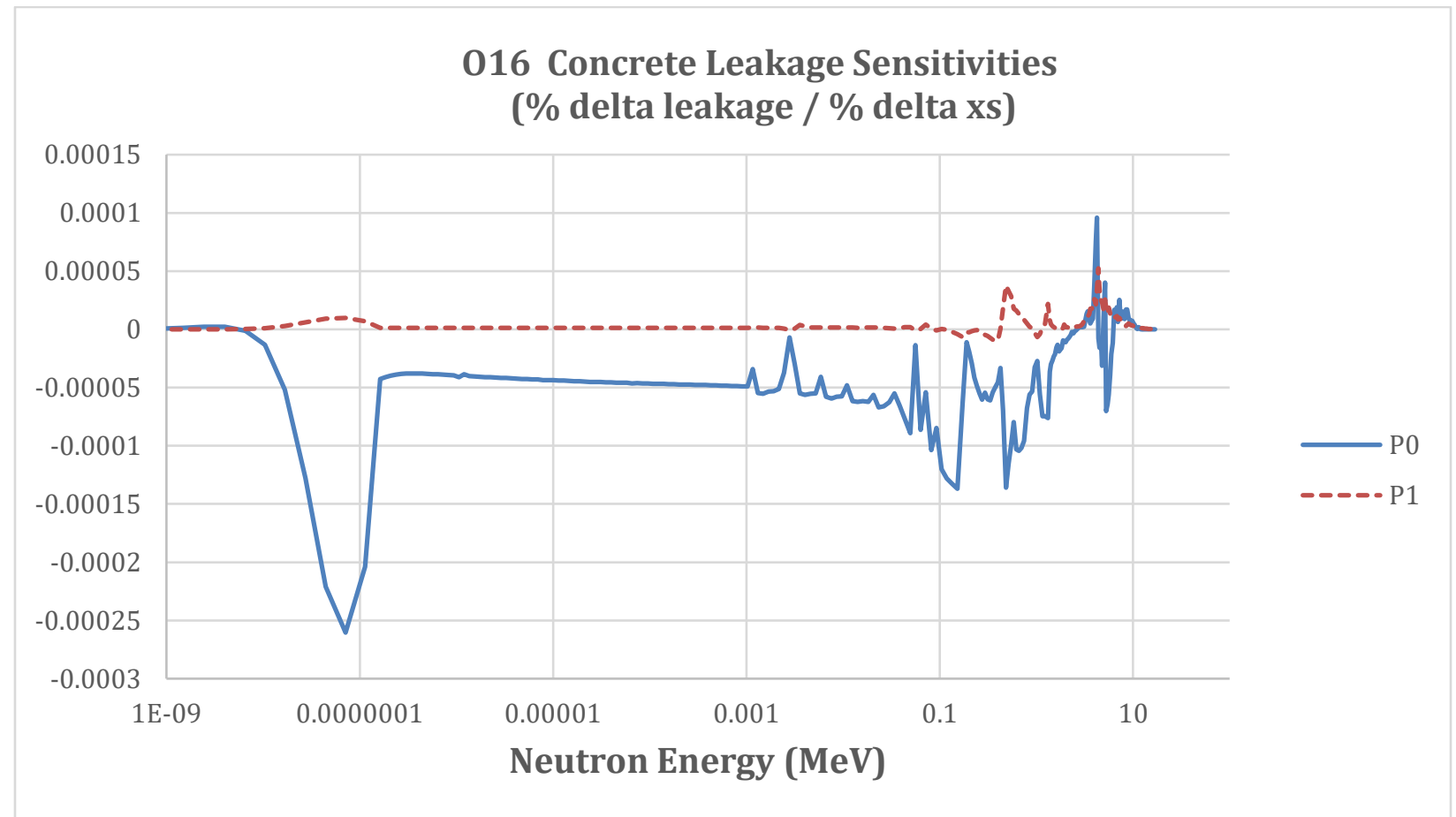

Figure 4. P0 and P1 Sensitivities for Leakage from the Concrete Sphere 


\subsection{Some 0-16 Results of mubar Sensitivity for the Concrete Sphere}

Using the JENDL 4.0 O-16 data, leakage sensitivity for the concrete sphere problem was calculated. Both the MT 2 elastic scattering and the MF 34 mubar scattering covariances were calculated with NJOY. The sensitivity vectors were taken from SENSMG calculations using ENDF/B VII.1 data. The actual values in Table II are of secondary concern - the important point is that the mubar uncertainty is comparable in magnitude to the elastic scattering uncertainty. Also note that there isn't much difference in the leakage sensitivity between the P0 sensitivity vector and the summed up total PL sensitivity vector.

Table II. Relative Leakage Uncertainty from the Concrete Sphere

\begin{tabular}{|c|c|c|c|}
\hline Covariance Data & Sensitivity Vectors & Number of Groups & Leakage Sens. (\%/\%) \\
\hline mt 2 JENDL 4.0 & P0 ENDF/B VII.1 & 250 & $8.701 \mathrm{e}-5$ \\
\hline $\mathrm{mt}$ 2 JENDL 4.0 & PL ENDF/B VII.1 & 250 & $8.710 \mathrm{e}-5$ \\
\hline $\mathrm{mf}$ 34 JENDL 4.0 & P1 ENDF/B VII.1 & 250 & $3.994 \mathrm{e}-5$ \\
\hline
\end{tabular}

\subsection{Some Pu-239 Results of mubar Sensitivity for Jezebel}

The ENDF/B VIII.0 evaluation for Pu-239 was also processed with NJOY to produce mubar covariances in 250 groups. These covariances were then used in the sandwich rule with sensitivities calculated by SENSMG for Jezebel. The sensitivities were calculated with ENDF/B VIII.0 data in a 1D discrete ordinates model. Thus, the result for the effect of mubar uncertainty on $\mathrm{k}_{\text {eff }}$ is directly comparable to the official sensitivity results for Jezebel given on Table VIII of the CIELO release paper ${ }^{9}$.

The only approximation is that the P1 sensitivities from SENSMG include inelastic scattering and $(\mathrm{n}, \mathrm{xn})$ reactions along with the elastic scattering. It is thus assumed that the elastic scattering is the dominant term in the P1 scattering block.

The uncertainties for Jezebel in the CIELO release paper range from 81 to $903 \mathrm{pcm}$ for the prompt fission neutron spectrum, the fission cross section, nubar, elastic and inelastic scattering, and capture for various cross section libraries including ENDF/B VIII.0 (called CIELO in the table). In Table III, mubar covariance results are given for Jezebel for comparison. ( $\mathrm{k}_{\mathrm{eff}}$ from SENSMG was 1.0006428.)

Table III. Jezebel Sensitivity to mubar Covariances

\begin{tabular}{|c|c|c|c|}
\hline Covariance Data & Sensitivity Vectors & Number of Groups & keff sensitivity (pcm) \\
\hline ENDF/B VIII.0 & P1 ENDF/B VIII.0 & 250 & 160.6 \\
\hline ENDF/B VIII.0 & P1 ENDF/B VII.1 & 250 & 160.3 \\
\hline
\end{tabular}

These results clearly show that mubar covariances are an important component of Jezebel $k_{\text {eff }}$ sensitivity, comparable to the other factors. In future Jezebel uncertainty analyses, mubar covariances should be included. 


\section{Generating Replica Data for Complex Dynamic Problems}

Replica data requires the generation of correlated random numbers. The idea is that the means, variances and covariance's of the replica data in aggregate should be equal to (or at least very close) to the means, variances and covariance's of the real data. As the number of replicas goes up, the replica data should match the real data more closely. However, if the number of replica data sets is limited, then the correlated random numbers may be pre-conditioned to match the real data exactly with respect to means, variances, and covariance's. This is the "empirical=' $T$ ' option of the mvrnorm function in " $R$ " - a widely used free software environment for statistical computing.

The desired output for the uncertainty propagation is the variance (or standard deviation) of the output quantity of interest. Since the variance is a higher order quantity than the mean, it takes more samples to achieve reasonable convergence. Thirty to fifty samples are usually enough for a mean, but at least seventy-five samples should be used to measure the uncertainty in the variance (or standard deviation).

When the individual uncertainty distributions are (or are assumed to be) Gaussian, then standard multi-variate normal random numbers may be used. When the individual uncertainty distributions are not Gaussian, then other methods such as copulas may be used to generate the correlated random samples. If the random samples may not be negative in value, then lognormal distributions can be used.

\subsection{Generating Replica NDI Cross Section Tables with mubar Covariance Data}

If mubar covariances are being represented by replica data, the replica data is entered into the multi-group cross section libraries as P1 elastic scattering components in the Legendre Scattering matrix for the isotope of interest. Care should be taken that the physical limits of -1 to +1 should not be exceeded for any group-wise value of mubar. Hence the ENDF/B VIII.0 O-16 covariance data cannot currently be used because of the very large uncertainties at specific high energy resonance points and also at lower energies.

When such replica data for O-16 from JENDL 4.0 has been used at Los Alamos, it has been found that the mubar uncertainties can be important for small reactor systems with high neutron leakage. In some cases, in fact, the sensitivity due to mubar uncertainty even exceeds the sensitivity due to the elastic scattering uncertainty.

\subsection{Summary}

Actual mubar covariances from evaluated nuclear data files have been applied to shielding and criticality problems. Consistent with previous studies, it has been found that small nuclear systems with lots of neutron leakage are sensitive to the uncertainties in mubar for $\mathrm{O}-16$ and $\mathrm{Pu}-$ 239. Mubar covariance data should be applied to all future sensitivity studies of small nuclear systems with a large fraction of neutron leakage. 


\section{References}

1. A. Trkov, M. Herman, and D.A. Brown, Editors, "ENDF-6 Formats Manual", CSEWG Document ENDF-102, National Nuclear Data Center, Brookhaven National Laboratory, (2012).

2. R. E. MacFarlane, "The NJOY Nuclear Data Processing System, Version 2012", LA-UR-1227079, Los Alamos National Laboratory, (2012).

3. X-5 Monte Carlo Team, "MCNP - A General Monte Caro N-Particle Transport Code, Version 5", LA-UR-03-1987 (revised 2008), Los Alamos National Laboratory, (2008).

4. J. A. Favorite, "SENSMG: First-Order Sensitivities of Neutron Reaction Rates, Reaction-Rate Ratios, Leakage, keff, and $\alpha$ Using PARTISN", Nuclear Science and Engineering, 192(1), pp. 80114, (2018).

5. G. Aliberti and R.D. McKnight, "Mu-bar Sensitivities", Transactions of the American Nuclear Society, San Diego, California, Nov. 11-15, 2012, Vol. 107, pp. 1093-1096, (2012).

6. B.C. Kiedrowski, "K-eigenvalue Sensitivity Coefficients to Legendre Scattering Moments", Transactions of the American Nuclear Society, Washington D.C., Nov. 10-14, 2013, Vol. 109, pp. 735-738, (2013).

7. M. Aufiero and M. Fratoni, "Testing Advanced Methods for Sensitivity/Uncertainty Analysis in the Monte Carlo Code SERPENT", PHYSOR 2016, Sun Valley, Idaho, May 1-5, 2016, pp.28942903, (2016).

8. J. A. Favorite, "Using the MCNP Taylor series perturbation feature (efficiently) for shielding problems", ICRS-13 \& RPSD-2016, EPJ Web of Conferences, 153, (2017).

9. M. B. Chadwick, et al, "CIELO Collaboration Summary Results: International Evaluations of Neutron Reactions on Uranium, Plutonium, Iron, Oxygen and Hydrogen", Nuclear Data Sheets, 148, pp. 189-213, (2018).

10. B. C. Kiedrowski, "MCNP6.1 k-Eigenvalue Sensitivity Capability: A User's Guide", MCNP Documentation \& Website, LA-UR-13-22251, Los Alamos National Laboratory, (2013).

11. B. C. Kiedrowski, "Importance of Scattering Distributions on Criticality", NCSD 2013, Nuclear Criticality Safety Division Topical Meeting, Wilmington, North Carolina, Sept. 29 - Oct 3, 2013. 